\title{
Medidas de funcionalidad en pacientes post- COVID atendidos en un hospital universitario de cuarto nivel. Análisis por ingreso a la unidad de cuidados intensivos
}

\section{Functional measures in post-Covid patients in a fourth- level university hospital: analysis by ICU admission}

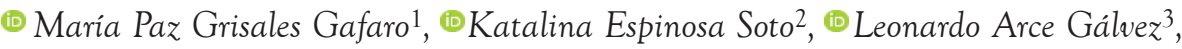
(D) María Ana Tovar Sánchez ${ }^{4}$

\section{RESUMEN}

Introducción. La infección por SARS-CoV2 genera una morbimortalidad importante, por lo cual es necesario conocer el estado funcional post infección de los pacientes con el fin de desarrollar intervenciones en rehabilitación adecuadas.

Objetivo. Definir el compromiso osteomuscular, funcional y de equilibrio en un grupo de pacientes recuperados de COVID-19 y atendidos en un hospital universitario de cuarto nivel de Cali, Colombia.

Materiales y métodos. Estudio descriptivo de corte transversal en el cual se realizaron mediciones funcionales en pacientes recuperados de COVID-19, los cuales se clasificaron en dos grupos: con ingreso o sin ingresó a la unidad de cuidados intensivos (UCI).

Resultados. En los pacientes con ingreso a UCI se encontró mayor número de comorbilidades y un mayor impacto funcional en escalas como la FIM, en especial el componente motor.

Conclusión. Dados los resultados, es de gran importancia hacerles un buen seguimiento a los pacientes sobrevivientes de COVID-19 para en el futuro diseñar planes específicos para su rehabilitación.

Palabras clave. Coronavirus, rehabilitación, cuidado intensivo.

\section{ABSTRACT}

Introduction. SARS-CoV2 infection generates significant morbidity and mortality, for this reason, it is necessary to know the post-infection functional status of the patients in order to develop appropriate rehabilitation interventions.

Objective. To define the musculoskeletal, functional, and balance compromise in a group of patients recovered from COVID-19 and treated in a fourth-level university hospital in Cali, Colombia.

Materials and methods. Descriptive cross-sectional study in which functional measurements were conducted in patients recovered from COVID-19 who were classified into two groups: those who were admitted to the intensive care unit (ICU) and those who were not.

Results. In the patients admitted to the ICU, a greater number of comorbidities were found, as well as a greater functional impact on scales such as the FIM, especially on the motor component.

Conclusion. Given the results, it is of great importance to follow-up closely the surviving COVID-19 patients in order to design specific plans for their rehabilitation in the future.

Keywords. Coronavirus, rehabilitation, intensive care.
Autores:

${ }^{1}$ Médico Residente. Departamento de Medicina Física y Rehabilitación, Universidad del Valle. Departamento de Medicina Física y Rehabilitación, Hospital Universitario del Valle Evaristo García E.S.E. Cali, Colombia.

${ }^{2}$ Médico Residente. Departamento de Medicina Física y Rehabilitación, Universidad del Valle. Departamento de Medicina Física y Rehabilitación, Hospital Universitario del Valle Evaristo García E.S.E. Cali, Colombia.

${ }^{3}$ Médico Residente. Departamento de Medicina Física y Rehabilitación, Universidad del Valle. Departamento de Medicina Física y Rehabilitación, Hospital Universitario del Valle Evaristo García E.S.E. Cali, Colombia.

${ }^{4}$ Médico Especialista. Departamento de Medicina Física y Rehabilitación, Universidad del Valle. Departamento de Medicina Física y Rehabilitación, Hospital Universitario del Valle Evaristo García E.S.E. Cali, Colombia.

Correspondencia:

Leonardo Arce Gálvez. Departamento de Medicina Física y Rehabilitación, Universidad del Valle, Hospital Universitario del Valle.

Calle 5 No. 36-08, edificio 112, Piso 1, Cali, Colombia. Correo electrónico: leonardo.arce@correounivalle.edu.co.

Recibido: 08.08.21

Aceptado: 26.01.22

\section{Citación:}

Grisales Gafaro MP, Espinosa Soto K, Arce Gálvez L, Tovar Sánchez MA.

Medidas de funcionalidad en pacientes post-COVID atendidos en un hospital universitario de cuarto nivel. Análisis por ingreso a la unidad de cuidados intensivos. Rev Col Med Fis Rehab 2021;31(2): 129-135

https://doi.org/10.28957/rcmfr.v31n2a3

Conflictos de interés:

Ninguno declarado por los autores. 


\section{INTRODUCCIÓN}

Tras la aparición en China de los primeros casos de infección por SARS-Cov2 en diciembre del 2019, el COVID-19, enfermedad causada por este nuevo coronavirus, se expandió rápidamente por todos los continentes al punto que el 11 de marzo de 2020 la Organización Mundial de la Salud ${ }^{1}$ la declaró una pandemia. Tal ha sido el alcance de esta entidad, que hasta el 31 diciembre de 2021 se habían registrado 285.530.130 casos y 5.426.781 muertes por esta causa en todo el mundo 2 .

El COVID-19 es un enfermedad grave de la cual se han realizado numerosos estudio; por ejemplo, Jin et al. ${ }^{3}$ establecieron que el 13,8\% de los pacientes presentan síntomas severos y el $4,7 \%$ requieren manejo en la unidad de cuidado intensivo (UCI). Lo anterior se debe a que la severidad clínica, el requerimiento de ventilación mecánica y la estancia hospitalaria son aspectos comunes en pacientes con esta enfermedad y que están relacionados directamente con el desarrollo del síndrome de distrés respiratorio agudo. Por otra parte, Meyer et al. ${ }^{4}$ encontraron que los hombres, los fumadores y los alcohólicos presentan un riesgo mayor de contagio, mientras que en mujeres, personas de estatura baja, diabéticos y afrodescendientes esta probabilidad es menos; sin embargo, en el caso de los pacientes afrodescendientes e hispanos la severidad puede ser mayor.

Asimismo, se ha establecido que las consecuencias del COVID-19 son trascendentes y perduran en el tiempo; según Fontela et al. ${ }^{5}$, de los pacientes que requieren ventilación mecánica en UCI por cualquier causa, entre el 25\% y el $50 \%$ desarrollan debilidad muscular y entre el $85 \%$ y el $90 \%$ persisten con debilidad por 2 a 5 años después del egreso hospitalario. De igual forma, otros autores han establecido que entre el $20 \%$ y el $30 \%$ persisten con deterioro cognitivo pasados dos años de la hospitalización y que además se espera que con la estancia hospitalaria prolongada la funcionalidad previa de los pacientes se deteriore tras el periodo de convalecencia ${ }^{6,7}$. Esto se ha evidenciado en algunos estudios que reportan puntajes bajos en escalas de funcionalidad tras la hospitalización por complicaciones cardiopulmonares, neurológicas y metabólicas ${ }^{8,9}$.

\section{MATERIALES Y MÉTODOS}

Estudio descriptivo transversal realizado para definir el compromiso osteomuscular, funcional y de equilibrio en un grupo de pacientes recuperados de COVID-19 mediante la prueba Timed Up and Go Test (TUGT). Esta prueba fue aplicada al momento del alta de los participantes, quienes fueron atendidos en un hospital universitario de alta complejidad de Cali, Colombia.

La funcionalidad de los participantes fue evaluada mediante escalas ampliamente conocidas: la medida de independencia funcional (FIM), que evalúa dos dimensiones (cognitiva y motor) mediante la valoración de 18 actividades (13 motoras y 5 cognitivas) ${ }^{10}$; el Manual Muscle Test (MMT), que evalúa la capacidad muscular en tres diferentes grupos osteomusculares ${ }^{11}$; la prueba TUGT, que evalúa la capacidad motriz a través de una prueba de cambio de posición y caminata corta $^{12}$, y la escala de funcionalidad postcovid (PCFS), que fue desarrollada durante la pandemia, evalúa la funcionalidad a través de una serie de preguntas sobre síntomas y dependencia, tiene una buena reproducibilidad, está siendo utilizada en distintos estudios alrededor del mundo y cuenta con una versión en español desarrollada para Colombia ${ }^{13}$.

\section{Análisis estadístico}

Las variables continuas se describieron mediante promedios y desviación estándar, o medianas y rangos intercuartiles, según la normalidad de los datos; la comparación entre estas variables se hizo mediante las pruebas T-student o U-Mann-Whitney. Por su parte, las variables categóricas se describieron mediante proporciones; la comparación entre estas variables se realizó con la prueba Chi-cuadrado o la prueba exacta de Fisher, según el caso. Se estableció un nivel de significancia estadística $a$ priori de $\alpha=0,05$. 


\section{CONSIDERACIONES ÉTICAS}

Los pacientes dieron su consentimiento para el uso de su información clínica y paraclínica y el estudio fue aprobado por los comités de ética de la Universidad del Valle y del Hospital Universitario del Valle.

\section{RESULTADOS}

Se incluyó un total de 108 participantes, de los cuales 88 habían ingresado a la UCI
(Tabla 1). No se encontró una diferencia estadísticamente significativa con relación al sexo, pero en los antecedentes personales sí se halló una relación estadísticamente significativa para el ingreso a UCI con los pacientes que padecían hipertensión u obesidad, o las dos condiciones. Los días de estancia hospitalaria fueron numéricamente superiores en los paciente no UCI, ya que estos ingresaron por motivos adicionales que debieron ser resueltos antes de su egreso; no hubo diferencia en relación con la intubación orotraqueal.

Tabla 1. Resultados de pacientes que ingresaron y no ingresaron a la unidad de cuidados intensivos.

\begin{tabular}{|c|c|c|c|c|c|c|}
\hline \multicolumn{3}{|c|}{ Variables } & $\begin{array}{c}\text { No UCI, } \\
n=20\end{array}$ & $\begin{array}{l}\text { UCI, } \\
\mathrm{n}=88\end{array}$ & $\begin{array}{c}\text { General, } \\
\mathrm{N}=108\end{array}$ & Valor $\mathrm{p}$ \\
\hline \multirow{2}{*}{ Sexo, n(\%) } & \multicolumn{2}{|c|}{ Masculino } & $14(70)$ & $44(50)$ & $58(53,7)$ & \multirow{2}{*}{0,17} \\
\hline & \multicolumn{2}{|c|}{ Femenino } & $6(30)$ & $44(50)$ & $50(46,3)$ & \\
\hline \multirow{6}{*}{$\begin{array}{l}\text { Antecedentes } \\
\text { clínicos, } n(\%)\end{array}$} & \multicolumn{2}{|c|}{ Comorbilidad } & $9(45)$ & $76(86,4)$ & $85(78,7)$ & 0,001 \\
\hline & \multicolumn{2}{|c|}{ Hipertensión } & $6(30)$ & $57(64,8)$ & $63(58,3)$ & 0,001 \\
\hline & \multicolumn{2}{|c|}{ Diabetes } & $4(20)$ & $33(37,5)$ & $37(34,3)$ & 0,21 \\
\hline & \multicolumn{2}{|c|}{ Obesidad } & $0(0)$ & $35(39,8)$ & $35(32,4)$ & 0,001 \\
\hline & \multicolumn{2}{|c|}{ Enfermedad pulmonar crónica } & $1(5)$ & $18(20,5)$ & $19(17,6)$ & 0,19 \\
\hline & \multicolumn{2}{|c|}{ Hipertensión y obesidad } & $0(0)$ & $25(28,4)$ & $25(23,1)$ & 0,001 \\
\hline \multicolumn{3}{|c|}{ Días hospitalizados, mediana (RIC) } & $40,0(28,0-58,75)$ & $29,0(16,75-49,0)$ & $30,0(19,0-52,25)$ & 0,03 \\
\hline \multicolumn{3}{|c|}{ Grado de la escala PCFS, mediana (RIC) } & $2,0(1,0-3,2)$ & $3,0(2,0-4,0)$ & $3,0(2,0-4,0)$ & 0,13 \\
\hline \multicolumn{3}{|c|}{ Intubación orotraqueal, n(\%) } & $\mathrm{Na}$ & $34(38,6)$ & $34(31,5)$ & - \\
\hline \multicolumn{3}{|c|}{ Realizar el ejercicio, $\mathbf{n}(\%)$} & $12(60)$ & $44(50)$ & $56(51,9)$ & 0,46 \\
\hline \multicolumn{3}{|c|}{ Tiempo en realizar el ejercicio, $n(\%)$} & $\mathrm{n}=12$ & $\mathrm{n}=44$ & $\mathrm{n}=56$ & \\
\hline \multicolumn{3}{|c|}{ de 0 a 10 segundos } & $2(16,7)$ & $6(13,6)$ & $8(14,3)$ & \multirow{3}{*}{0,53} \\
\hline \multicolumn{3}{|c|}{ De 11 a 15 segundos } & $5(41,7)$ & $12(27,3)$ & $17(30,4)$ & \\
\hline \multicolumn{3}{|c|}{ Mayor a 15 segundos } & $5(41,7)$ & $26(59,1)$ & $31(55,4)$ & \\
\hline \multicolumn{3}{|c|}{ Tiempo en realizar el ejercicio, mediano (RIC) } & $14,5(11-18,2)$ & $17,0(12,7-22,0)$ & $16,0(12,0-21,2)$ & 0,31 \\
\hline \multirow{12}{*}{\multicolumn{2}{|c|}{$\begin{array}{l}\text { Dominios evaluados, } \\
\text { mediana (RIC) }\end{array}$}} & Dominio motor & $81,5(55,2-90,2)$ & $68(36,7-88)$ & $72(37-89)$ & 0,19 \\
\hline & & Dominio cognitivo & $35(29,5-35)$ & $35(28,7-35)$ & $35(28,7-35)$ & 0,94 \\
\hline & & Autocuidado & $40,5(27,2-42,0)$ & $36(18-42)$ & $37(18,7-42)$ & 0,46 \\
\hline & & Movilidad & $16,5(12-21)$ & $14(3,75-21)$ & $15(4-21)$ & 0,25 \\
\hline & & Ambulación & $10,5(4,2-13,2)$ & $8(2-12)$ & $8(2-12)$ & 0,2 \\
\hline & & Comunicación & $14(11,5-14)$ & $14(12-14)$ & $14(12-14)$ & 0,87 \\
\hline & & Control de Esfínteres & $14(9-14)$ & $14(8,7-14)$ & $14(8,75-14)$ & 0,49 \\
\hline & & Conocimiento Social & $21(18-21)$ & $21(17-21)$ & $21(17-21)$ & 0,79 \\
\hline & & Escala FIM & $116,5(81,5-125,2)$ & $103(66,5-123)$ & $106(67,7-123,2)$ & 0,27 \\
\hline & & Flexores de codo & $5(4-5)$ & $5(4-5)$ & $5(4-5)$ & 0,46 \\
\hline & & Flexores de cadera & $5(4-5)$ & $5(4-5)$ & $5(4-5)$ & 0,7 \\
\hline & & Flexores de rodilla & $5(4-5)$ & $5(4-5)$ & $5(4-5)$ & 0,65 \\
\hline
\end{tabular}

Fuente: elaboración propia. 
En la prueba de TUGT no se encontró una diferencia significativa; sin embargo, el número de pacientes que tardó más de 15 segundos en completarla fue superior en el grupo de ingreso a UCI. En la escala FIM se encontró un puntaje total más bajo en el grupo de ingreso a UCI, siendo más representativo en el dominio motor. La valoración de fuerza del MMT en determinados grupos musculares no mostró una diferencia estadísticamente significativa.

\section{DISCUSIÓN}

La inactividad física, secundaria a hospitalizaciones prolongadas, trae como consecuencia un desacondicionamiento físico que afecta particularmente a los huesos, los músculos y el sistema cardiovascular, lo que a su vez genera un importante impacto en la independencia en las actividades de la vida diaria y, en consecuencia, un deterioro en la calidad de vida ${ }^{14}$. Tal falta de actividad física es mayor en pacientes hospitalizados en la UCI, por lo que es usual que en esta población aumente la debilidad muscular y el delirio, y se prolongue el tiempo con ventilación mecánica ${ }^{15}$.

En el contexto de la pandemia por COVID19 se han desarrollado publicaciones que describen las complicaciones que ocasiona la enfermedad en la condición física en relación con el estado crítico de los pacientes; estas complicaciones se caracterizan por el inicio agudo de un desacondicionamiento físico y la pérdida de la fuerza y la masa muscular, por un lado, y el desarrollo difuso de miopatías y neuropatías, por el otro ${ }^{16}$.

En su estudio, Candan et al. ${ }^{17}$ establecieron que entre el $8 \%$ y el $30 \%$ de los pacientes con COVID-19 tienen pérdida de masa muscular en los primeros 7-10 días posteriores al ingreso a la UCI, lo cual limita la funcionalidad de las personas a su egreso; esto contrasta con los resultados del presente estudio, en donde el componente motor de la FIM tuvo una disminución estadísticamente significativa, pero el MMT en la valoración clínica no presentó diferencias. La prueba de equilibrio y funcionalidad TUGT, el componente cognitivo de la FIM y la PCFS tampoco presentaron diferencias estadísticamente significativas, al igual que no lo hicieron la diabetes mellitus y la enfermedad pulmonar crónica, resultados que difieren a los reportados en estudios simulares ${ }^{18}$.

El síndrome de atención post intensiva incluye una serie de trastornos que abarcan el deterioro físico, cognitivo y de la salud mental; este surge después de una enfermedad crítica y persiste más allá de la atención aguda hospitalaria, por lo que genera impacto en la calidad de vida de los pacientes ${ }^{18}$. Por lo anterior, con el fin de prevenir y mitigar consecuencias físicas, cognitivas y emocionales, es de vital importancia el inicio rápido de la rehabilitación ${ }^{19,20}$. Asimismo, es indispensable tener presente que la disminución de la capacidad funcional ocasionada por este síndrome genera un aumento considerable de la demanda de los servicios de rehabilitación para limitar las consecuencias económicas relacionadas con la pérdida de la capacidad funcional ${ }^{6}$.

Como fortaleza del presente estudio se tiene que es una de las primeras mediciones funcionales posterior a la infección por Covid-19 en Colombia y Latinoamérica. Como dificultades resaltan, por un lado, la diferencia en el número de pacientes con ingreso a UCI en comparación con los que no tuvieron ingreso a dicha unidad, esto debido a la situación clínica particular de los pacientes en la institución del estudio, y, por el otro, el hecho de que no se consideraron factores adicionales como el tipo de plan de rehabilitación y la intensidad del mismo, lo que puede generar una modificación en el estado funcional del paciente.

\section{CONCLUSIÓN}

El estado post infeccioso por COVID-19 en el paciente con comorbilidades que requieren estancia hospitalaria en UCI genera una dependencia funcional parcial, especialmente en el dominio motor, pero también con un 
compromiso menor del dominio cognitivo, aunque esto no tiene una relación directa según el MMT; asimismo, se encontró un incremento en el tiempo requerido para realizar el TUGT en los pacientes con ingreso a UCI. Dado este panorama, es de gran importancia hacerles un buen seguimiento a los pacientes sobrevivientes de COVID-19 para en el futuro diseñar planes específicos para su rehabilitación.

\section{FINANCIACIÓN}

Ninguna declarada por los autores.

\section{RESPONSABILIDADES ÉTICAS}

\section{Protección de personas y animales}

Los autores declaran que para esta investigación no se han realizado experimentos en seres humanos ni en animales.

\section{Confidencialidad de los datos}

Los autores declaran que han seguido los protocolos de su centro de trabajo sobre la publicación de datos de pacientes.

\section{Derecho a la privacidad y consentimiento informado}

Los autores declaran que en este artículo no aparecen datos que puedan identificar a las personas que respondieron la encuesta; así mismo, se contó con la aprobación de los encuestados para divulgar los datos.

\section{CONTRIBUCIÓN DE LOS AUTORES}

María Paz Grisales Gafaro y Katalina Espinosa Soto estuvieron a cargo de la revisión de bibliografía, el protocolo de investigación, la recolección de los datos, el análisis de resultados y la redacción del manuscrito; Leonado Arce Gálvez se encargó de la revisión de bibliografía, el protocolo de investigación, la recolección de los datos, el análisis de resultados, la redacción del manuscrito y el sometimiento a la revista, y María Ana Tovar Sánchez se ocupó del protocolo de investigación, el análisis de los resultados y la redacción del manuscrito. 


\section{REFERENCIAS}

1. Pérez-Campos Mayoral L, Hernández-Huerta MT, Mayoral-Andrade G, Pérez-Campos Mayoral E, Pérez-Campos E. A letter to the editor on "World Health Organization declares global emergency: A review of the 2019 novel Coronavirus (COVID-19)." Int J Surg. 2020;79:163-4. Disponible en: https://doi.org/10.1016/j.ijsu.2020.05.066.

2. Colombia. Ministerio de Salud y Protección Social (Minsalud). Coronavirus (COVID-19). Bogotá D.C.: Minsalud; 2021 [citado diciembre 31 de 2021]. Disponible en: https:// www.minsalud.gov.co/salud/publica/PET/Paginas/Covid-19_copia.aspx.

3. Jin Y, Yang H, Ji W, Wu W, Chen S, Zhang W, et al. Virology, Epidemiology, Pathogenesis, and Control of COVID-19. Viruses. 2020;12(4):-372. Disponible en: https://doi.org/10.3390/v12040372.

4. Meyer NJ, Gattinoni L, Calfee CS. Acute respiratory distress syndrome. Lancet. 2021;398(10300): 622-37. Disponible en: https://doi.org/10.1016/S0140-6736(21)00439-6.

5. Fontela PC, Abdala FANB, Forgiarini SGI, Forgiarini LA. Quality of life in survivors after a period of hospitalization in the intensive care unit: A systematic review. Rev Bras Ter Intensiva. 2018;30(4):496-507. Disponible en: https://doi.org/10.5935/0103-507X.20180071.

6. Stam HJ, Stucki G, Bickenbach J. Covid-19 and post intensive care syndrome: A call for action. J Rehabil Med. 2020;52(4):19-22. Disponible en: https://doi.org/10.2340/16501977-2677.

7. Choon-Huat Koh G, Hoenig H. How Should the Rehabilitation Community Prepare for 2019. nCoV? Arch Phys Med Rehabil. 2020;101(6):1068-71. Disponible en: https://doi.org/10.1016/j.apmr.2020.03.003.

8. Liu K, Zhang W, Yang Y, Zhang J, Li Y, Chen Y. Respiratory rehabilitation in elderly patients with COVID-19: A randomized controlled study. Complement Ther Clin Pract. 2020;39:101166. Disponible en: https://doi.org/10.1016/j.ctcp.2020.101166.

9. Vallejo-Serna R, Cantor-González JF, Arce-Gálvez L. Síndrome de Guillain-Barré asociado a COVID-19: diagnóstico, tratamiento y rehabilitación. Neurol Perspect 2021;1(1):104-6. Disponible en: https://doi.org/10.1016/j.neurop.2021.03.003.

10. Graham JE, Granger CV, Karmarkar AM, Deutsch A, Niewczyk P, Divita MA, et al. The uniform data system for medical rehabilitation: Report of follow-up information on patients discharged from inpatient rehabilitation programs in 2002-2010. Am J Phys Med Rehabil. 2014;93(3):231-44. Disponible en: https://doi.org/10.1097/PHM.0b013e3182a92c58.

11. Fan E, Ciesla ND, Truong AD, Bhoopathi V, Zeger SL, Needham DM. Inter-rater reliability of manual muscle strength testing in ICU survivors and simulated patients. Intensive Care Med. 2010;36(6):1038-43. Disponible en: https://doi.org/10.1007/s00134-010-1796-6.

12. Mathias S, Nayak US, Isaacs B. Balance in elderly patients: the "get-up and go" test. Arch Phys Med Rehabil. 1986;67(6):387-9.

13. Siegerink B, Boon D, Barco S, Klok E, Le J. The Post-COVID-19 Functional Status (PCFS) Scale: a tool to measure functional status over time after COVID-19. OSF; 2022. Disponible en: https://doi.org/10.17605/OSF.IO/QGPDV.

14. Kramer A, Gollhofer A, Armbrecht G, Felsenberg D, Gruber M. How to prevent the detrimental effects of two months of bed-rest on muscle, bone and cardiovascular system: An RCT. Sci Rep. 2017;7(1):1-10. Disponible en: https://doi.org/10.1038/s41598-017-13659-8. 
15. Nydahl P, Sricharoenchai T, Chandra S, Kundt FS, Huang M, Fischill M, et al. Safety of patient mobilization and rehabilitation in the intensive care unit: Systematic review with meta-analysis. Ann Am Thorac Soc. 2017;14(5):766-77. Disponible en: https://doi.org/10.1513/AnnalsATS.201611-843SR.

16. Ahmad I, Rathore FA. Neurological manifestations and complications of COVID-19: A literature review. J Clin Neurosci. 2020;77:8-12. Disponible en: https://doi.org/10.1016/j.jocn.2020.05.017.

17. Candan SA, Elibol N, Abdullahi A. Consideration of prevention and management of long-term consequences of post-acute respiratory distress syndrome in patients with COVID-19. Physiother Theory Pract. 2020;36(6):663-8. Disponible en: https://doi.org/10.1080/09593985.2020.1766181.

18. Smith JM, Lee AC, Zeleznik H, Coffey-Scott JP, Fatima A, Needham DM, et al. Home and Community-Based Physical Therapist Management of Adults With Post-Intensive Care Syndrome. Phys Ther. 2020;100(7):1062-73. Disponible en: https://doi.org/10.1093/ptj/pzaa059.

19. Fuke R, Hifumi T, Kondo Y, Hatakeyama J, Takei T, Yamakawa K, et al. Early rehabilitation to prevent postintensive care syndrome in patients with critical illness: A systematic review and metaanalysis. BMJ Open. 2018;8(5):1-10. Disponible en: https://doi.org/10.1136/bmjopen-2017-019998.

20. Needham DM, Davidson J, Cohen H, Hopkins RO, Weinert C, Wunsch H, et al. Improving longterm outcomes after discharge from intensive care unit: Report from a stakeholders' conference. Crit Care Med. 2012;40(2):502-9. Disponible en: https://doi.org/10.1097/CCM.0b013e318232da75. 